\title{
Contenido de sodio en pan elaborado en panaderia de distribucion nacional y comparado con una panadería local en la ciudad de Chillán de Chile
}

\author{
Comparison of sodium content in bread \\ from a bakery with national \\ distribution and a local bakery \\ of Chillán city, Chile
}

\begin{abstract}
Introduction: The Chilean Ministry of Health (MINSAL) and the Chilean Federation of Bakers (FECHIPAN) agreed to progressively decrease sodium content in bread from 800 to $500 \mathrm{mg} / 100 \mathrm{~g}$ bread in 100 bakeries in 2011 and in 100\% of bakeries in 2014 . Objective: To analyze and compare the sodium content in bread from a bakery with national distribution (A) and a local bakery (B). Materials and methods: A total of 100 samples were analyzed in each bakery, 50 each of the two types of bread known as marraqueta and hallulla (types of bread rolls). Analysis was performed at different times of the day and at different days of the week. Sodium content was determined by the AOAC (2005) method using atomic absorption spectrophotometry and compared with the sodium content declared in the nutrition label in bakery $A$. Differences between content for type of bread, bakery, and time of sampling were established by ANOVA and Student's t-test with the STATA 12.0 software at a $p<0.05$ level of significance. Results: Total sodium in bread ( $\mathrm{mg} / 100 \mathrm{~g}$ unit) was estimated as $619.6 \pm 127.7 \mathrm{mg}$ for hallulla and $641.0 \pm 93.3$ for marraqueta. There were no significant differences in sodium content in both bakeries $(p=0.971)$ and type of bread $(p=0.177)$. Sodium content was higher on Wednesdays $(p<0.0001, p=0.016)$ and at 17 hours $(p<0.000001, p=0.028)$ in hallulla and marraqueta, respectively. Total sodium content in both bakeries varied between 412.5 and 954.5 mg/100 g. Conclusions: Bread from both bakeries showed similar sodium contents although they exceeded the MINSALFECHIPAN agreement by $26 \%$ to $80 \%$.

Key words: Bread, sodium, bakeries.
\end{abstract}

\section{INTRODUCCIÓN}

Las enfermedades cardiovasculares (ECV) constituyen la principal causa de muerte a nivel mundial y han sido catalogadas como una pandemia por la Organización Mundial de la Salud (OMS) (1). El Informe del Estado Global en Salud del año 2010 (2), señala que las ECV causaron aproximadamente el 63\% (36 millones) de las muertes ocurridas en el mundo durante ese año, y que la mortalidad aumentará en $17 \%$ en los próximos 10 años (3).

En las últimas décadas, Chile ha experimentado una no-
Ximena Aguilera P. (1)

Camila López S. (1)

Alejandra Rodriguez F. (1)

Ximena Sanhueza R. (1)

Eduardo Atalah S. (2)

1Julio Parra F. (1)

(1) Departamento de Nutrición y Salud Pública, Facultad Ciencias de la Salud y de los Alimentos, Universidad del Bío-Bío, Chillán, Chile. (2) Departamento de Nutrición, Facultad de Medicina,

Universidad de Chile. Santiago, Chile.

Dirigir la correspondencia a: Dr. Julio Parra Flores Nutricionista Magister en Salud Pública Departamento Nutrición y Salud Pública Universidad del Bío-Bío, sede Chillán CP 3780000 Chile Tel: (56)42-2463107 - Fax 42-2463132 Email: juparra@ubiobio.cl

Este trabajo fue recibido el 14 de Enero de 2014 y aceptado para ser publicado el 10 de Mayo de 2014.

toria transición epidemiológica, demográfica y nutricional, con un aumento de las expectativas de vida de los chilenos, pero con un incremento de las ECV, siendo la hipertensión arterial un factor relevante como causa primaria (4).

La importancia de la hipertensión arterial (HA) como problema de salud pública radica en su rol causal de morbimortalidad cardiovascular. La presión arterial tiene una relación muy estrecha, continua y graduada con el desarrollo de enfermedades cardiovasculares, infarto agudo del miocardio (IAM), insuficiencia renal, enfermedad arterial periférica y 
todas las causas de muerte cardiovascular (5).

La presión arterial se encuentra íntimamente ligada a la ingesta de sodio, el cual es esencial para nuestro cuerpo en pequeñas cantidades, participa en la mantención de la homeostasis de los diferentes fluidos del organismo y en las neuronas en las génesis de estímulos eléctricos, que centralmente regulan el funcionamiento de nuestro organismo (6). Sin embargo, su ingesta en altas cantidades está asociado al incremento de la presión arterial y a problemas generales de salud cardiovascular (7). Por lo que la restricción del sodio en estos casos constituye una medida básica dentro del esquema terapéutico de la hipertensión.

La cantidad de sodio requerida para un adulto se estima en unos 500 miligramos (1,25 gramos de $\mathrm{NaCl}$ ) al día. Sin embargo, la ingesta normal de sodio en forma de cloruro de sodio varía de 2-14 gramos (0,8 - 5,6 gramos de sodio) al día (8). En Chile, según la Encuesta Nacional de Salud 2009-2010, la población chilena tiene un consumo diario de 9,8 gramos por persona al día (9).

En el 2009, la OPS y la OMS impulsaron la iniciativa sobre la reducción del consumo de sal en el continente americano, con el propósito de prevenir las ECV. Uno de los objetivos principales era revisar las políticas y programas regionales existentes con el fin de emitir recomendaciones para reducir gradualmente el consumo de sal en la dieta y alcanzar las metas internacionalmente recomendadas de un valor inferior a 5 gramos de sal por persona al día para el 2020. Debido a esto, diferentes países del mundo, y también Chile, están implementando actualmente programas para reducir el consumo de sal/sodio en la población.

Recientes estudios plantean que la mayoría de los alimentos procesados y de alto consumo presentan concentraciones elevadas de sodio (10). El pan no es la excepción. El pan está clasificado como uno de los alimentos de mayor consumo en el mundo y ser una elevada fuente de sodio dietario (11), siendo responsable en promedio del 30\% de la ingesta de sal (12).

En Chile, según cifras entregadas por Euromonitor Internacional, nuestro país lidera el consumo de pan per cápita en Latinoamérica con alrededor de $95 \mathrm{~kg} / \mathrm{habitante}$ al año siendo el tercer país después de Alemania con 106 kg (13) y Turquía de $150 \mathrm{~kg} /$ habitante por año respectivamente (14).

En un estudio realizado el 2010 por el Ministerio de Salud de Chile en relación al contenido de sodio en 52 muestras de 4 variedades de pan (marraqueta, hallullas, frica y baguette) de la Región Metropolitana, mostró una concentración promedio de $774 \pm 105 \mathrm{mg} / 100$, con un nivel mayor en las marraquetas $(831 \pm 105 \mathrm{mg} / 100 \mathrm{~g})$ en relación a las hallullas $(735 \pm 92 \mathrm{mg} / 100 \mathrm{~g})$. Por lo que en Septiembre del
2010, el MINSAL junto con la Federación Gremial Chilena de Industriales Panaderos (FECHIPAN) y la Asociación Chilena de Supermercados (ASACH) llegaron a un acuerdo para reducir la sal en la fabricación de este alimento en los próximos 4 años. Dentro de este acuerdo se estableció como meta para los tipos de pan de consumo más común (hallulla y marraqueta) disminuir aproximadamente de 800 a $500 \mathrm{mg}$ de sodio (2 a $1,25 \mathrm{~g} \mathrm{NaCl}$ ) por $100 \mathrm{~g}$ de pan para diciembre del 2011 y a 400 $\mathrm{mg}$ de sodio $(1 \mathrm{~g} \mathrm{NaCl})$ para diciembre del 2014. Esta iniciativa comenzó con un programa piloto que incluyó 100 panaderías a lo largo del país, con el compromiso de incorporar al menos 500 panaderías para fines del 2011 y al 100\% de las empresas asociadas para el 2014 (15).

El objetivo de este estudio fue analizar el contenido de sodio en el pan hallulla y marraqueta elaborada en un supermercado de distribución nacional y compararla con el producido en una panadería local en el contexto del acuerdo nacional de reducción de sodio.

\section{MATERIALES Y MÉTODOS}

Se utilizó un estudio analítico de corte transversal en las 2 panaderías con la mayor producción en Chillán (sobre 1000 kg diarios de pan).

Muestreo: De cada panadería se analizó un total de 100 muestras, de las cuales 50 eran pan tipo marraqueta y 50 tipo hallulla. La panadería A (supermercado), utiliza una premezcla de preparación estandarizada y que es distribuida nacionalmente, y la panadería B (local), una mezcla de ingredientes de confección diaria. Las muestras fueron obtenidas a diferentes horas del día $(9: 00 ; 12: 00 ; 17: 00$ y 20:00 h) y en distinto días de la semana (lunes a viernes).

Análisis de sodio: La concentración de sodio en el pan se determinó en el laboratorio acreditado de experimentación y certificación de alimentos (LECYCA) de la Universidad del Bío-Bío, utilizando el método oficial 985.35 AOAC (2005) mediante un espectrofotómetro de absorción atómica. Este método consiste en secar el producto hasta obtener sus cenizas, diluirlas en ácido Clorhídrico (37\%) y luego realizar una curva de calibración/concentración/absorbancia con un estándar para ser leída a una longitud de onda de 589 nm (16). Además, se comparó el contenido de sodio declarado en la etiqueta de pan hallulla y marraqueta (453 y $451 \mathrm{mg} / 100 \mathrm{~g}$ pan) de la panadería A con lo obtenido para cada tipo de pan en el análisis de laboratorio. Los resultados se expresaron en $\mathrm{mg}$ de sodio/100 g pan y se realizaron por duplicados.

Análisis de los datos: Se confeccionó una base de datos en Microsoft Excel 2010. Se describieron las variables a través de la estadística descriptiva. Se calcularon frecuencias absolutas

TABLA 1

Contenido total de sodio (mg/100 g pan) según panadería y tipo de pan.

\begin{tabular}{|c|c|c|c|c|c|c|c|}
\hline & \multicolumn{2}{|c|}{ Panadería A } & \multicolumn{2}{|c|}{ Panadería B } & \multicolumn{2}{|c|}{ Total } & \multirow[t]{2}{*}{$\mathrm{p}$} \\
\hline & $x$ & D.E & $\mathrm{x}$ & D.E & $x$ & D.E & \\
\hline Hallulla & 618,9 & 122,6 & 620,3 & 113,7 & 619,6 & 127,7 & 0,956 \\
\hline Marraqueta & 641,2 & 113,1 & 640,9 & 69,3 & 641,1 & 93,3 & 0,982 \\
\hline Total & 630,0 & 117,9 & 630,6 & 106,5 & 630,3 & 112,1 & 0,177 \\
\hline $\mathrm{p}$ & \multicolumn{2}{|c|}{0,347} & \multicolumn{2}{|c|}{0,337} & \multicolumn{2}{|c|}{0,971} & \\
\hline
\end{tabular}

T-student 
y porcentajes, promedio y desviación estándar según el tipo de variables. Se utilizó la prueba de ANOVA y de T-student para establecer si existían diferencias en la cuantificación del sodio por tipo de pan, hora de extracción y lugar de expendio. Se utilizó el software STATA 12.0 con un nivel de significancia de estadística $\alpha 0,05$.

\section{RESULTADOS}

El contenido promedio de sodio total en ambas panaderías (A y B) fue de 630,2 $\$ 112,0 \mathrm{mg} / 100 \mathrm{~g}$ de pan, no existiendo diferencias significativas entre la panadería $A$ y $B(p=0,971)$. Con respecto al pan hallulla, la panadería $A$ presenta una media de $618,8 \pm 122.6 \mathrm{mg}$ y en la panadería B una media de $620,3 \pm 133,7$, no siendo diferente entre ambos lugares de venta $(p=0,956)$. El pan marraqueta en la panadería $A$ presenta $641,2 \pm 113,1$ y la panadería B $640,8 \pm 69,3$, siendo el contenido de sodio significativamente similar en ambos tipo de pan ( $p=0,988$ ) (tabla 1$)$.

El 92\% de las muestras de pan de la Panadería A y el $77 \%$ de la panadería $B$, presentan valores de sodio superiores a $500 \mathrm{mg} / 100 \mathrm{~g}$ pan.

Al evaluar el contenido de sodio en el pan según día de la semana, el día miércoles fue significativamente mayor el contenido de sodio en el pan marraqueta $(p=0.028)$ y en el pan hallulla $(p<0,00001)$ (tabla 2$)$.

Con respecto a la hora de extracción de las muestras, el contenido de sodio fue significativamente mayor a las $17: 00$ tanto para el pan marraqueta $(p=0.028)$ y pan hallulla $(p<0,00001)$ (tabla 3).

Al evaluar y comparar el contenido de sodio del pan hallulla y marraqueta con su etiquetado nutricional en la panadería A, el 99\% de las muestras de pan no cumplían con lo declarado. (figura 1).

\section{DISCUSIÓN}

El 2006 la OMS y la OPS realizaron el foro sobre la "Reducción del Consumo de Sal en la Población", con expertos de varios países. En dicho foro, las recomendaciones para las naciones se plantearon en torno a tres pilares fundamentales: a) reformulación de los productos alimenticios con participación de la industria del rubro; b) concientización y educación en el consumidor sobre etiquetado nutricional y c) efectos nocivos del sodio y cambios ambientales para hacer más accesible hábitos saludables (17).

Un informe técnico posterior elaborado por la OMS y la Organización de las Naciones Unidas para la Agricultura y la Alimentación (FAO), recomendaba el consumo de menos de $2 \mathrm{~g}$ de sodio (5 gramos de sal) por día como meta de ingesta de nutrientes de la población, garantizando al mismo tiempo su yodación. Esta consulta hizo hincapié en que la ingesta alimentaria de sodio procedente de todas las fuentes influye en los niveles de presión arterial de la población y que de-

\section{TABLA 2}

Contenido de sodio por tipo de pan (mg/100 g de pan) según hora de extracción.

\begin{tabular}{lcccc}
\hline & \multicolumn{2}{c}{ Pan hallulla } & \multicolumn{2}{c}{ Pan marraqueta } \\
Hora & $\mathrm{n}$ & Media* & $\mathrm{n}$ & Media* $^{*}$ \\
09:00 & 40 & $577,0 \pm 123,5$ & 40 & $637,6 \pm 99,0$ \\
$12: 00$ & 20 & $583,8 \pm 53,8$ & 20 & $601,2 \pm 60,3$ \\
$17: 00$ & 20 & $755,0 \pm 95,1$ & 20 & $688,6 \pm 111,1$ \\
20:00 & 20 & $604,8 \pm 130,1$ & 20 & $639,8 \pm 71,9$ \\
p & & 0,000001 & & 0,028 \\
\hline${ }^{* \text { media+ds. }}$ ANOVA & & & \\
\hline
\end{tabular}

TABLA 3

Contenido de sodio en ambos tipos de pan (mg/100 g de pan) según hora de extracción.

\begin{tabular}{lcccc}
\hline & \multicolumn{2}{c}{ Pan hallulla } & \multicolumn{2}{c}{ Pan marraqueta } \\
Día & $\mathrm{n}$ & Media* & 20 & \multicolumn{1}{c}{ Media* } \\
Lunes & 20 & $541,5 \pm 127,2$ & 20 & $601,2 \pm 50,3$ \\
Martes & 20 & $583,8 \pm 53,8$ & 20 & $688,6 \pm 111,1$ \\
Miércoles & 20 & $755,0 \pm 95,1$ & 20 & $639,8 \pm 71,9$ \\
Jueves & 20 & $604,8 \pm 130,1$ & 20 & $662,8 \pm 126,0$ \\
Viernes & 20 & $612,6 \pm 111,6$ & & 0,016 \\
p & & 0,0001 & & \\
\hline *media+ds. & & & \\
ANOVA & &
\end{tabular}


bía limitarse su uso para lograr reducir el riesgo de padecer cardiopatías coronarias y accidentes cerebrovasculares (18).

Numerosos estudios han establecido que la mayor parte del sodio ingerido por un individuo es aportado por los alimentos elaborados industrialmente. En Chile, se estima que $75 \%$ del sodio presente en la dieta se añade a los alimentos durante su procesamiento o manufactura; y sólo 10\% procede de los alimentos naturales. La sal es utilizada como preservante y acentuante del sabor en innumerables alimentos, por lo que es ampliamente utilizada por la industria alimentaria especialmente en la industria panadera (19).

Estudios internacionales han indicado buenos resultados de aceptabilidad del sabor del pan con disminuciones paulatinas de la sal en su fabricación (20), sin embargo la relación aceptabilidad del consumidor vs inocuidad del producto aún no ha sido estudiada. Un aspecto tampoco estudiado son los aditivos utilizados en la preparación de pan con alto contenido de sodio.

Recientemente, Valenzuela y cols (2013) evaluaron el programa piloto de reducción de sodio/sal en 10 panaderías adheridas y 10 no adheridas de la Región Metropolitana de Chile, no encontrando diferencias significativas en la contenido promedio de sodio en ambas panaderías. Sin embargo existía bastante variabilidad en los niveles de sodio en ambos grupos, con valores extremos de 403 y 824 mg/100 g. Concluyendo que la concentración de sodio en el pan fue similar en ambos grupos y que existe preocupación de la industria panadera por un pan más saludable.

En nuestro estudio, también encontramos que no había diferencias significativas en el contenido de sodio por tipo de pan y tipo de panadería, pero que no cumplían el acuerdo de reducción de sodio. Preocupante son los valores encontrados en la panadería $A$, la cual no estaría cumpliendo con el etiquetado nutricional que ellos declaran que sería menor a $500 \mathrm{mg}$ de sodio en $100 \mathrm{~g}$ de pan. Esta empresa informa en su publicidad mediante diferentes medios comunicación un pan bajo en sodio, lo que puede influir en la elección de dicho expendio por sobre otros por parte del consumidor. $\mathrm{Si}$ consideramos que en la elaboración del pan se utiliza una mezcla estandarizada para todo el país, entonces podemos concluir que a nivel nacional no se cumple con la declaración en el etiquetado nutricional y tampoco con un pan más saludable bajo en sodio.

El año 2010 se implementó en Argentina el programa "Menos Sal, Mas Vida" con más de 6000 panaderías adheridas, logrando una reducción de $25 \%$ de sal en la fabricación del pan. En una evaluación realizada como parte de este programa, en la provincia de la Pampa, se encontró diferencia estadísticamente significativa en la sal utilizada tanto en la fabricación del pan como de otros productos de pastelería entre los años 2010 y 2011 (21).

En Australia entre el 2007-2010 la proporción de panes con un contenido de sodio menor a 400 mg/100 g aumentó de $25 \%$ a $50 \%$. En Nueva Zelanda en el mismo período el aumento fue $0 \%$ a 17\% (22). En el Reino Unido por su parte, informa al año 2010 un contenido de sodio en el pan blanco de 397 mg/100 g/pan (23). La Unión Europea, en tanto, se ha comprometido a reducir durante los años 2009-2013 en 16\% la cantidad de sal en todas las categorías de alimentos (24).

Aun cuando estas iniciativas son exitosas, es necesario evaluar su aplicabilidad en nuestro país, debido al fuerte componente cultural asociado al consumo de pan en la población.

\section{FIGURA 1}

Diferencias en el contenido de sodio declarado en etiquetado nutricional por tipo de pan de la panadería A.

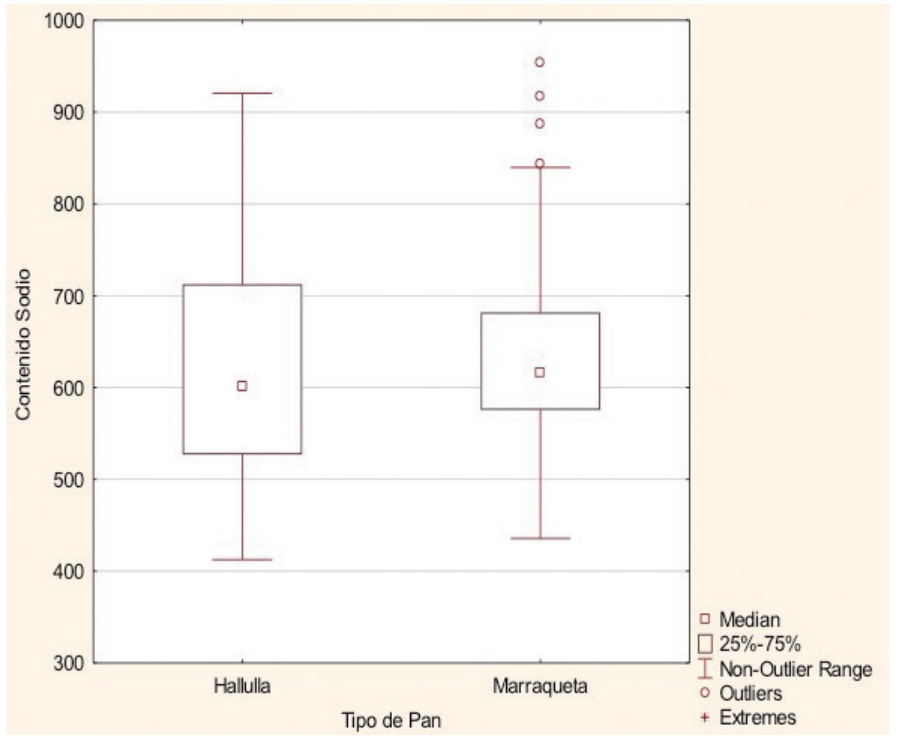

T-student

---- Contenido de sodio declarado en etiqueta nutricional 
El gusto por lo salado y por lo dulce son gustos adquiridos: mientras más azúcar o más sal se le agrega a la comida, mayor cantidad de azúcar y sal se le tendrá que poner a lo largo del tiempo para sentir el mismo gusto. Si se quita, el 50\% del azúcar o de la sal que tienen los productos que comemos habitualmente, se sentirá que no tienen sabor, que son insípidos y habrá un rechazo al ingerirlos (25).

La panadería B es una panadería tradicional para el consumidor de Chillán y distribuye a muchos otros expendios de pan en la ciudad y en la provincia de Ñuble. En ella, el contenido de sodio es similar a la panadería $A$, no existiendo diferencias para el consumidor cuando compra pan de acuerdo al contenido de sodio, pero si en relación al costo del producto ya que es más barato. No podemos establecer el porqué de las diferencias en los contenidos de sodio en algunos días y horas específicos, pero estas situaciones deben ser evaluadas y analizadas al interior de las panaderías a fin de establecer estrategias para evitar estas situaciones.

Aun cuando no existen diferencias significativas en el contenido de sodio entre ambos tipos de pan ( $p>0.05)$, no deja de ser alarmante que entre una hallulla y otra, presenten valores tan diversos llegando a 920,5 mg de sodio, lo que equivale a 2,3 gramos de sal. En caso de la marraqueta el resultado no es diferente ya que se alcanzan valores máximos de 954,5 con una equivalencia de 2,4 g aproximadamente, lo que en la práctica a veces es la recomendación diaria para una persona con HTA para todo el día. Si la OMS recomienda $5 \mathrm{~g}$ de sal al día o $2 \mathrm{~g}$ de sodio (26), con sólo consumir 100 g de pan (2 porciones diarias), estamos cubriendo el $120 \%$ del requerimiento diario de este mineral, sin tomar en cuenta el sodio contenido propio de los alimentos y de los aditivos.

Si consideramos que $26,9 \%$ de la población chilena y 29,2\% en la Región del Bío-Bío ( $3^{\circ}$ más alta del país) tienen hipertensión arterial (9), un alto consumo de este mineral en la dieta afectará directamente su función renal al desequilibrar la capacidad de concentración y eliminación de sodio por el riñón y agravar sus niveles de HTA.

Se puede decir que en general, la cantidad de sodio contenida en el pan, ya sea de panaderías o envasado no está siendo fiscalizado, ya que no existe ninguna norma que los obligue a tener un máximo de este mineral, sólo un plan piloto por parte del gobierno en el que están algunas panaderías y supermercados del país que se adhirieron voluntariamente en 2 años a reducir gradualmente la cantidad de sodio.

Este tema posee una importancia a nivel de Nutrición en Salud Pública, en donde los profesionales nutricionistas y de salud en general, deben contar con la información exacta al entregar recomendaciones alimentarias. Además, de que la población debe estar cabalmente informada y capacitada en la lectura de la información nutricional.

En conclusión, cuatro aspectos son importantes de considerar: a) el alto contenido de sodio en el pan estudiado; b) el incumplimiento del etiquetado por parte de una de las empresas y su publicidad errónea a la población; c) el poco control de la autoridad de salud y poca difusión de la estrategia de reducción de sodio en el pan y d) mientras el sistema no esté regulado y fiscalizado, la reducción de la sal en el pan sólo quedará a la voluntad de los empresarios.

\section{RESUMEN}

Introducción: El Ministerio de Salud de Chile (MINSAL) y la Federación de Panaderos de Chile (FECHIPAN) acordaron reducir progresivamente el contenido de sodio en el pan de 800 mg a 500 mg/100 g pan, en 100 panaderías el 2011 y en
100\% de ellos el año 2014. Objetivo: Analizar el contenido de sodio en pan de una panadería de distribución nacional (A) comparada con una de tipo local (B). Materiales y métodos: Se analizaron 100 muestras de pan por panadería, 50 de marraqueta y 50 de hallulla, en diferentes horas y días de la semana. El contenido de sodio se determinó con el método AOAC (2005) mediante espectrofotometría de absorción atómica y se comparó con el contenido de sodio declarado en el etiquetado nutricional de la panadería A. Se utilizó ANOVA y T-student para establecer diferencias del contenido de sodio por tipo de pan, panadería y hora de extracción, utilizando el software STATA 12.0 con un nivel de significancia $\mathrm{p}<0,05$. Resultados: El sodio total en el pan ( $\mathrm{mg} / 100 \mathrm{~g}$ pan) se estimó en $619,6 \pm 127,7 \mathrm{mg}$ para hallulla y $641 \pm 93,3$ para marraqueta, sin diferencias significativas para su contenido en ambas panaderías $(p=0,971)$ y tipo de pan $(p=0,177)$. El sodio fue mayor el día miércoles $(p<0,0001-p=0.016)$ y a las 17 horas $(p<0,000001-p=0.028)$ en pan hallulla y marraqueta respectivamente. Con variabilidad en el contenido de sodio total en ambas panaderías entre 412,5 a 954,5 mg / $100 \mathrm{~g}$. Conclusiones: El pan de ambas panaderías presenta similar contenido de sodio pero excediéndose $26 \%$ a $80 \%$ del acuerdo MINSAL-FECHIPAN.

Palabras clave: Pan, sodio, panaderías.

Agradecimientos: Al laboratorio de experimentación y certificación de alimentos (LECYCA) de la Universidad del Bío-Bío, a la Dirección de Escuela de la Carrera de Nutrición y Dietética, Dirección del Departamento de Nutrición y Salud Pública y la subdirección de Desarrollo Estudiantil de la Universidad del Bío-Bío para el logro de la ejecución de este proyecto.

\section{BIBLIOGRAFÍA}

1. Ministerio de Salud de Chile. Reporte de Vigilancia de Enfermedades No Transmisibles (ENT). Situación Epidemiológica de las ENT en Chile. Unidad de Estudios y Vigilancia de Enfermedades No Transmisibles. Departamento de Epidemiología. División de Planificación Sanitaria, Subsecretaría de Salud Pública, 2011:4-6.

2. World Health Organization. Global status report on non communicable diseases 2010. <http://www.who.int/nmh/ publications/ncd_report_full_en.pdf> [25-11-2013].

3. World Health Organization: Action Plan for the Global Strategy for the Prevention and Control of Noncommunicable Diseases 2008. <http://www. who.int/nmh/publications/9789241597418/en/index.htm/> [25-11-2013].

4. Crovetto M., Durán M., Guzmán M. Estudio descriptivo de la frecuencia y duración de la publicidad alimentaria emitida en la programación de canales de televisión asociados a anatel. Rev Chil Nutr. 2011; 38:290-9.

5. Ministerio de Salud de Chile. Guía Clínica Hipertensión Arterial Primaria o esencial en personas de 15 años y más, Santiago. 2006.

6. Monckeberg F. La sal es indispensable para la vida ¿pero cuánta? Rev Chil Nutr. 2012; 39 (4):192-5.

7. Jimenez-Maroto L, Sato T, Rankin S. Saltiness potentiaton in White bread by substituting sodium chloride with a fermented soy ingredent. J. Cereal Sci. 2013; 58:313-7.

8. Wardlaw GM. Perspectives of nutrition. 4 ed. New York, USA. Mc Graw Hill International. 1993; pp 472-92.

9. Encuesta Nacional de Salud 2009-2010. Ministerio de Salud de Chile. Pág. 1- 1064. Disponible: http://web.minsal.cl/ portal/url/item/bcb03d7bc28b64dfe040010165012d23. pdf. Consultado 08/05/2014. 
10. Belz M, Ryan L, Arendt E. The impact of salt reduction in bread: A review. Crit Rev Food Sci Nutr. 2012; 52:514-24.

11. Greenfield H, Smith A, Maple J, Wills R. Contributions af foods to sodium in the Australian food-supply. Hum NutrAppl Nutr. 1984; 38(2):203-10.

12. Girgis S, Neal B, Prescott L, Prendergast J, Dumbrell S, Turner C, Woodward M. A one quarter reduction in the salt content of bread can be made without detection. Eur J Clin Nutr. 2003; 57(4):616-20.

13. Estudio Chile Saludable: oportunidades y desafíos de innovación. Volumen II. Pág.1-37. Disponible en: http:// www.eligevivirsano.cl/wp-content/uploads/2013/05/ ESTUDIO-CHILE-SALUDABLE-VOLUMEN-II.pdf. Obtenida de Euromonitor Internacional. Fecha consulta: 08/05/2014.

14. Quilez J, Salas J. Salt in bread in Europe: potential benefits of reduction. Nutr Rev. 2012; 70(11):666-78.

15. Valenzuela K, Quitral V, Villanueva B, Zavala F, Atalah E. Evaluación del programa piloto de reducción sal/sodio en el pan en Santiago de Chile. Rev Chil Nutr. 2013; 40(2):119-22.

16. A.O.A.C. Official Methods of Analysis of Official Analytical Chemist. 18th Edition. Horwitz W. \& Latimer G.W. Editors. U.S.A., 2005.

17. Organización Panamericana de la Salud. Iniciativa sobre la reducción de la sal en las Américas: Hoja informativa. <http://new.paho.org/hq/dmdocuments/2009/sal_ini_ hoja_inf.pdf> [27-06-2013].

18. Organización Mundial de la Salud: Reducción del consumo de sal en la población <http://www.who.int/dietphysicalactivity/salt-report-SP.pdf> [10-06-2013].

19. Saieh C., Lagomarsino E. Hipertensión arterial y consumo de sal en pediatría. Rev Méd Chil. 2009; 80:11-20.

20. Girgis S, Neal B, Prescott J, Prendergast J, Dumbrell S, Turner $C$, Woodward M. A one-quarter reduction in the salt content of bread can be made without detection. Eur J Clin Nutr. 2003; 57(4):616-20.

21. Vázquez M, Lema S, Contarini A, Kenten C. Sal y salud, el punto de vista del consumidor Argentino obtenido por la técnica de grupos focales. Rev Chil Nutr. 2012; 39(4): 185-90.

22. Dunford EK, Eyles $H$, Mhurchu CN, Webster JL, Neal BC. Changes in the sodium content of bread in Australia and New Zealand between 2007 and 2010: implications for policy. Med J Aust. 2011; 195(6):346-9.

23. Webster JL, Dunford EK, Neal BC. A systematic survey of the sodium contents of processed foods. Am J Clin Nutr. 2010; 91(2):413-2.

24. Barbosa C, Boro M Pacheco da Costa $R$, Araújo A. Development of a method for controlling salt and sodium use during meal preparation for food services. Rev Nutr Campinas 2013; 26(1):75-87.

25. Instituto Nacional de Tecnología Industrial (INTI): Campaña Menos Sal, Más Vida. Disponible en: http://www.inti.gov. ar/sabercomo/sc93/inti4.php

26. Hiddo J, Heerspink L, Navis G, Ritz E. Salt intake in kidney disease- a missed therapeutic opportunity?. Nephrol Dial Transplant. 2012; 27:3435-42. 